\title{
On the role of distortion in the hcp vs fcc competition in rare-gas solids
}

\author{
N.V. Krainyukova \\ B. Verkin Institute for Low Temperature Physics and Engineering of the National Academy of Sciences of Ukraine \\ 47 Lenin Ave., Kharkov 61103, Ukraine \\ E-mail: ninakrai@yahoo.com \\ Received December 10, 2010
}

\begin{abstract}
As a prototype of initial or intermediate structure somewhere in between the hcp and fcc lattices we consider a distorted bcc crystal. We calculate the temperature and pressure dependences of the lattice parameters for heavier rare gas solids $\mathrm{Ar}, \mathrm{Kr}, \mathrm{Xe}$ in the quasiharmonic approximation applying the Aziz potentials and confirm that in line with the previously found prevalence of hcp over fcc the hcp structure is still dominant in the bulk over the wide $P-T$ ranges analyzed. The situation is different for confined clusters up to $10^{5}$ atoms where owing to the specific surface energetics and terminations the structures with five-fold symmetry comprising fcc fragments are dominant. As a next step we consider a free relaxation of differently distorted bcc clusters, and show that two types (monoclinic and orthorhombic) of the initial distortion and its degree is a driving force for the hcp vs fcc final realizations. Possible energetic links between the initial and final structures are shown and analyzed.
\end{abstract}

PACS: 61.50 .Ah Theory of crystal structure, crystal symmetry; calculations and modeling; crystal growth;

61.50.Ks Crystallographic aspects of phase transformations; pressure effects;

64.70.K- Solid-solid transitions;

71.15.Nc Total energy and cohesive energy calculations.

Keywords: rare-gas solids, phase transformations, lattice distortion.

\section{Introduction}

The problem of the hep vs fcc competition in rare gas solids (RGS) has a long history [1]. Only some specific theories based on a first-principle local density approximation $[2,3]$ or density-functional perturbation theory [4] confirm that the fcc structure may be expected at ambient pressures (in line with experiment) but it is still unclear why the theories based on the pair potentials like LennardJones (LJ) predict that the hep structure is the only possibility while experimental observations evidence in favor of the absolutely dominant fcc structure. Some certain progress is attained accounting not only two- but also manybody interactions $[1,5,6]$. Alternatively the explanation of such a discrepancy based on the specific surface energetics and terminations elaborated in particular in our works $[7,8]$, which show that the structures with five-fold symmetry comprising fcc fragments are dominant up to $10^{5}$ atoms, is also valid. The transformations of the fcc structure towards the hep meet large barriers, therefore for larger sizes they are impeded. Within last two and half decades several experimental groups observed the transition from the fcc to the hep structure in RGS under high pressures [9-12]. These observations have in particular revealed some features, which are not typical for usual structural phase transitions. One is the sluggishness of such transformations, which stretch within several dozens GPa, the other is a persistence of such transitions until their completion. The purpose of our work is to make the bulkrelated calculations within a relatively wide $P-T$ range (in order to frame some important trends) applying more realistic Aziz potentials, to analyze the mechanisms of the fcc-hcp transformations elaborating some new ideas and to discuss the possibilities of their experimental confirmation.

\section{The energetics of rare-gas solids}

It is well known that for the LJ potential the hcp is more favorable than the fcc by $\sim 0.01 \%$ [1]. We try to analyze the same with the Aziz pair potential:

$$
\begin{aligned}
& U(R)=\sum_{\infty}\left[\exp \left(\alpha-\beta R-\gamma R^{2}\right)-f_{c}(R) \sum_{6,8,10} C_{n} R^{-n}\right], \\
& f_{c}(R)=\left\{\begin{array}{c}
\exp \left[-\left(r^{*} / R-1\right)^{2}, R<r^{*}\right. \\
1, R>r^{*},
\end{array}\right.
\end{aligned}
$$


here $R$ is an interatomic distance, $\alpha, \beta, \gamma, C_{n}$ and $r^{*}$ are constants, which can be found for $\mathrm{Ar}, \mathrm{Kr}$ in [13] and for $\mathrm{Xe}$ in [14]. We consider the free energy (per atom):

$$
F=U(r)+F_{\text {kin }}+P V,
$$

where the potential energy $U(r)$ is calculated applying the Aziz potential and a summation runs over the infinite crystal; $P, V$ and $T$ are pressure, volume (per atom) and temperature respectively. We are searching for a minimum of $F$. The kinetic energy $F_{\text {kin }}$ is calculated within the quasiharmonic approach by analogy with [15] applying the similar method, i.e.,

$$
F_{\text {kin }}=\frac{3 \Theta}{2} \int_{0}^{1} \omega \rho(\omega) \mathrm{d} \omega+3 T \int_{0}^{1} \ln \left[1-\exp \left(-\frac{\omega \Theta}{T}\right)\right] \rho(\omega) \mathrm{d} \omega .
$$

In [15] it was shown that the Debye formula can be successfully replaced for numerous applications by the Einstein approach:

$$
F_{\text {kin }}=\frac{3 \Theta}{2}+3 T \ln \left[1-\exp \left(-\frac{\Theta}{T}\right)\right] .
$$

As $\Theta$ we used $\Theta^{2} \sim R^{-2} d / d R\left(R^{2} d U / d R\right)$, i.e., we calculate $\Theta$ in a quasiharmonic approach suggested by LJ and Devonshire [16]. For the $\Theta$ calculations we used the experimental $\Theta$ values [17] as references, which were found at ambient pressures and $T$ approaching zero. The free energy (1) is a function of two variables $P, T$ and one parameter $V$; the latter can be easily expressed via the nearest neighbor distance $R_{\mathrm{NN}}$ for both fcc and hep lattices. The validity of our method is confirmed by a good agreement of the calculated and experimental nearest neighbor distances as functions of temperature (Fig. 1). These calculations in particular showed that the LJ potential with the improved

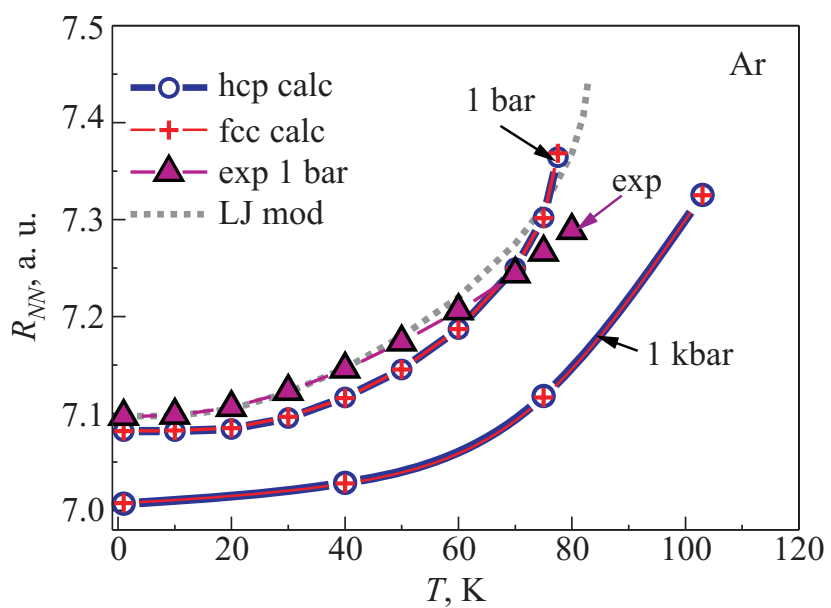

Fig. 1. (Color online) Temperature dependences of calculated (this work) and experimental [17] nearest neighbor distances for Ar at 1 bar. The inset shows the structure identifications. For comparison we show the calculated values for $1 \mathrm{kbar}$. The dotted line presents the calculation performed for the LJ potential with improved parameters [15].
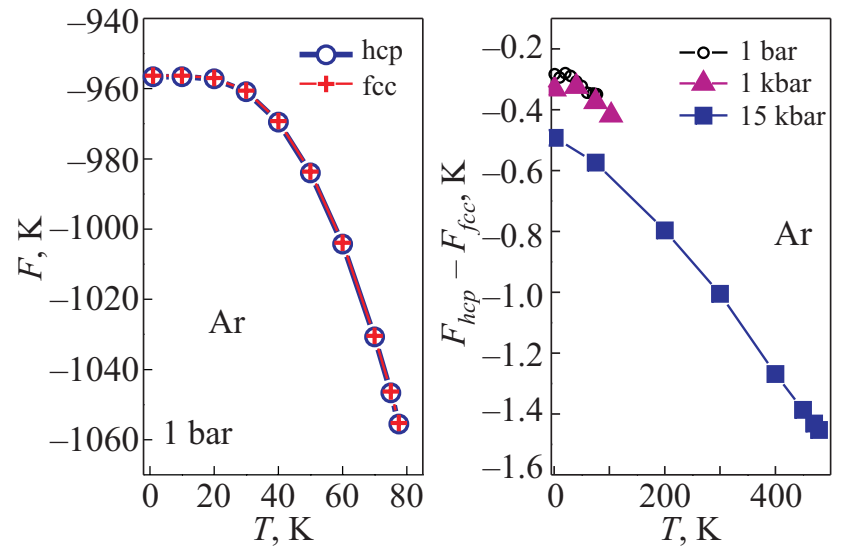

Fig. 2. (Color online) The temperature dependences of the free energy $F$ and the difference $F$ between the hcp and the fcc (in favor of the hcp) for Ar. The insets show the structure and pressure identifications.
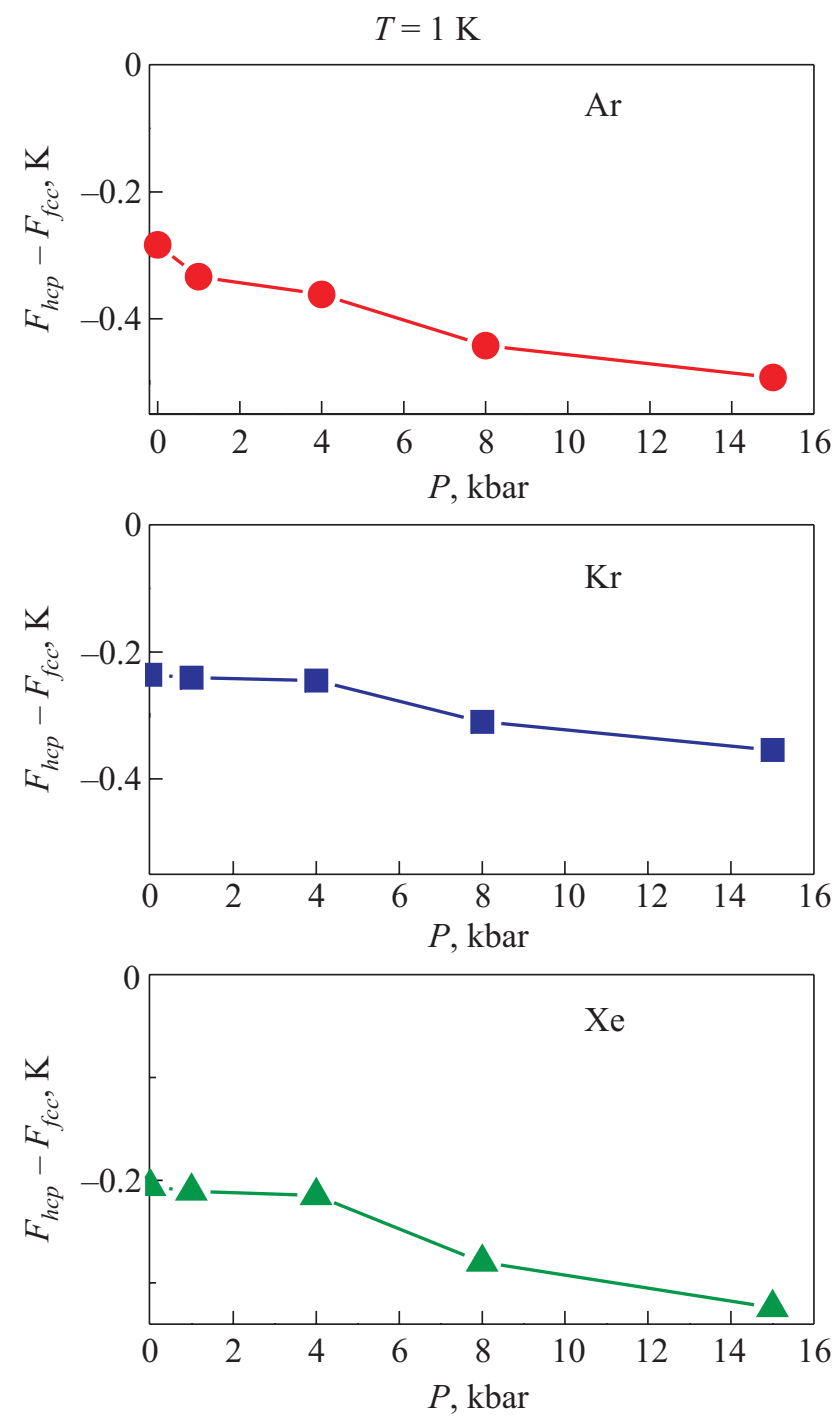

Fig. 3. (Color online) The difference $F$ between the hcp and the fcc (in favor of the hcp) as a function of pressure for $\mathrm{Ar}, \mathrm{Kr}$ and Xe. 
parameters (adjusted to the experimental data under study [15]) produces also a rather good coincidence with experiment.

The next figure (Fig. 2) demonstrates the temperature dependences of the free energy and the difference $F$ between hcp and fcc lattices at various pressures. Each dependence terminates at the point where the second derivative of the free energy with respect to interatomic distances attains zero, i.e., the crystal becomes unstable at such a point. We should mention here that the $\Theta$ values at relevant temperatures are still positive and sufficiently large. We can see from these dependences that the difference between the hcp and the fcc (in favor of the hcp) is not reduced with temperature but grows up. The similar effect is observed for the increasing pressure (Fig. 3) for all solid rare gases. These results confirm a growing gain of the hcp at elevated pressures in line with the experimental observations [9-12] but do not explain the observation of the fcc at ambient pressures. We assume that it can be due to a sizedependent effect mentioned above $[7,8]$ that was confirmed in particular in our previous studies of the size dependence of the formed structures in impurity-helium solids [18] but apparently other reasons may not be excluded.

\section{Structural transformations}

The next and important question is: how to observe the fcc-hcp transformation and is it possible at all because barriers are large? The relevant mechanisms were studied within a first-principle local density approximation [2] where two paths were suggested for an explanation of unusual features typical for the fcc-to-hcp phase transition (see Introduction). At lower pressures authors suggest the sliding-like scheme but at growing $P$ they assume that the intermediate face-centered orthorhombic (fco) structure may be responsible for the transformation mechanism because according to their calculations this phase becomes more favorable than the fcc at some pressure. As an argument in favor of this concept they calculated the barriers for the sliding mechanism and showed that such barriers crucially increase with $P$. Unfortunately they have not calculated the barriers for the fcc-fco transition, and as it will be shown below these barriers also significantly increase with $P$.

We have found previously that the bcc crystallites may freely relax towards the hcp structure under the applied LJ and Aziz potentials [19,20]. In some particular distortion cases an increasing contribution of the fcc phase was found [20]. More specifically the fraction of the fcc component in a final (after relaxation) crystal gradually increases if we expand the crystal along $c$ (Fig. 4, orthorhombic distortion). A similar result can be attained if we gradually reduce the angle between $a$ and $b$ from $90^{\circ}$ to $70.5^{\circ}$ (Fig. 4, monoclinic distortion). As we can see in the lower panels of Fig. 4 in both cases the crystal after allowed relaxation

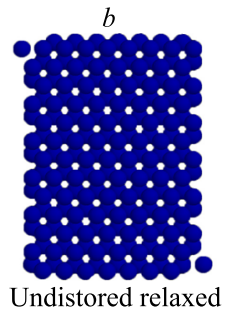

(hcp)

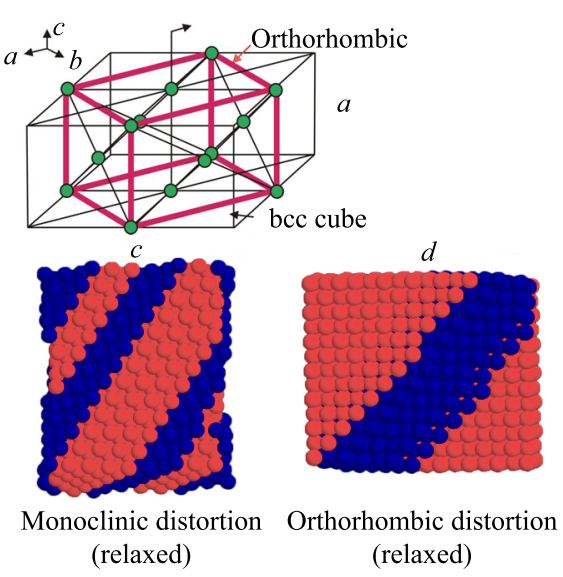

Fig. 4. (Color online) A general scheme, this describes mutual transformations fcc-bcc-hcp. The orthorhombic cell is cut from 4 bcc cubes. For the hep lattice the cell is rectangular, $c$ coincides with $a_{h c p}, b$ coincides with $c_{h c p}$ and every 2 nd (010) plane is displaced by $a / 6$; for the fcc lattice $a=b=c=a_{f c c}$ or the same fcc lattice can be obtained if we reduce the angle between $a$ and $b$ from $90^{\circ}$ to $70.5^{\circ}$ and transform all parameters in such a way that the plane (010) will be a basal plane $(a)$. The results of a free relaxation of the undistorted bcc lattice ( $b$, the final hep structure) and of the bcc distorted in monoclinic $(c)$ and orthorhombic $(d)$ ways. As dark atoms we show atoms with the hep-like environment, light gray atoms have the fcc-like environment.

is enriched with stacking faults (SF). This finding implies that in a real experiment both orthorhombic and monoclinic distortions result after relaxation in abundances of $\mathrm{SF}$ that make them undistinguishable from each other.

We calculated also barriers, which prevent the fcc-hcp transformation for both monoclinic and orthorhombic distortions (the top panels in Fig. 5). We see that for the orthorhombic distortion barriers are not lower than for the monoclinic one and grow up with pressure that does not support the main argument in favor of two paths between the fcc and hcp structures at increasing pressures suggested in [2] and discussed at the beginning of this section. But apparently even more important is the other result shown in the lower panels of Fig. 5. We analyzed the volume relaxation under both distortions and have found that the volume grows up for the monoclinic distortion. It implies in particular that if the transformation fcc-hcp is initiated at growing pressures it can result in the 'negative' compressibility that is impossible. Therefore the monoclinic distortion is realistic only at decompression (if the fcc-hcp transformation is invertible). In contrast with the monoclinic distortion the orthorhombic one (in a path from the fcc) results in the lattice compression that is consistent with an increasing $P$ (which is necessary for the transition observation according to experiment). Moreover this finding may explain the sluggishness of the fcc-hcp transition, because the additional pressure is needed to compensate the volume reduction. The estimations show that in the 

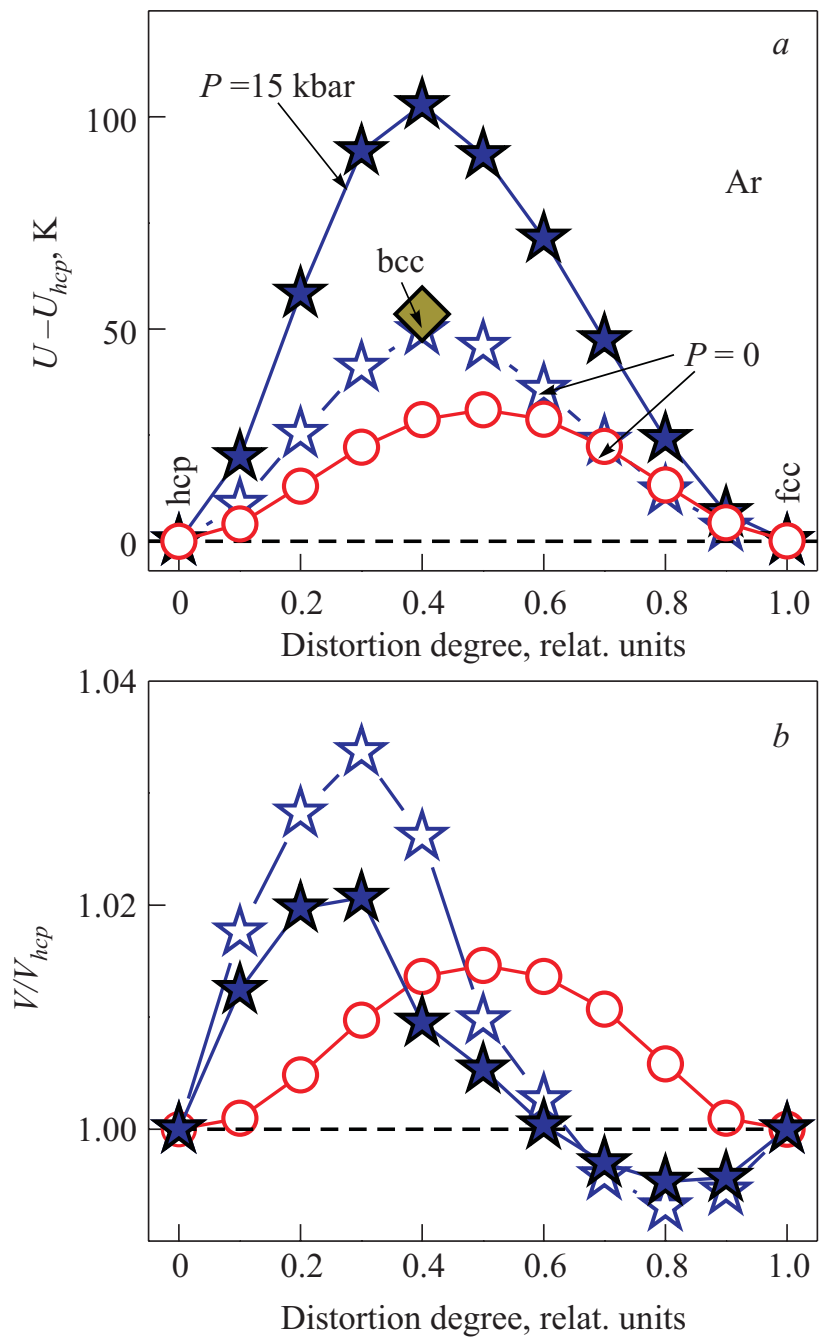

Fig. 5. (Color online) Calculated barriers for the monoclinic and orthorhombic distortions (see the figure for identifications). The calculation for the pure bec crystal at $P=0$ is shown as a diamond $(a)$. The volume variations are shown in the panels $(b)$. Orthorhombic distortion ( $\star$ ) and ( $(\downarrow)$, monoclinic distortion $(O)$.

point of the minimum of $V / V_{h c p}$ (Fig. 5) at increasing pressures, $P \Delta V$ ( $\Delta V$ is negative) can be close to the relevant barrier that facilitates the transformation.

\section{Summary}

Applying the Aziz potential we show that the competition between hcp and fcc is still in favor of hcp (as for the LJ potential), the difference increases with $T$ and growing pressures. A size-dependent energetic prevalence of fcc is likely an important reason for the mentioned dominant observation of fcc in RGS although other reasons may not be excluded. As concerns the mechanism of the possible fcc-hcp transformation we show that the known scheme of sliding along the basal planes is plausible only for decompression while at growing pressures we suggest as much more probable an alternative possibility when the bcc distorted in a orthorhombic way participates as a third counterparty in such a transformation.

The author is grateful to D. Lee, M.A. Strzhemechny, Yu.A. Freiman, V. Kiryukhin, and V. Khmelenko for fruitful discussion of this work.

1. Rare Gas Solids, M.L. Klein and J.A. Venables (eds.), Academic Press, London (1976), Vol. 1.

2. E. Kim and M. Nicol, Phys. Rev. Lett. 96, 035504 (2006).

3. I. Kwon, L.A. Collins, J.D. Kress, and N. Troullier, Phys. Rev. B52, 15165 (1995).

4. J.K. Dewhurst, R. Ahuja, S. Li, and B. Johansson, Phys. Rev. Lett. 88, 075504 (2002).

5. Yu. Freiman and S.M. Tretyak, Fiz. Nizk. Temp. 33, 719 (2007) [Low Temp. Phys. 33, 545 (2007)].

6. Yu. Freiman, A.F. Goncharov, S.M. Tretyak, A. Grechnev, J.S. Tse, D. Errandonea, H.-k. Mao, and R. Hemley, Phys. Rev. B78, 014301 (2008).

7. N.V. Krainyukova, Thin Solid Films 515, 1658 (2006).

8. N.V. Krainyukova, Eur. Phys. J. D43, 45 (2007) (and Refs. therein).

9. A.P. Jepcoat, H.-k. Mao, L.W. Finger, D.E. Cox, R.J. Hemley, and C.-S. Zha, Phys. Rev. Lett. 59, 2670 (1987).

10. H. Cynn, C.S. Yoo, B. Baer, V. Iota-Herbei, A.K. McMahan, M. Nicol, and S. Carlson, Phys. Rev. Lett. 86, 4552 (2001).

11. D. Errandonea, B. Schwager, R. Boehler, and M. Ross, Phys. Rev. B65, 214110 (2002).

12. D. Errandonea, R. Boehler, S. Japel, M. Mezouar, and L.R. Benedetti, Phys. Rev. B73, 092106 (2006).

13. R.A. Aziz and M.J. Slaman, Molec. Phys. 58, 679 (1986).

14. R.A. Aziz and M.J. Slaman, Molec. Phys. 57, 825 (1986).

15. N.V. Krainyukova, Fiz. Nizk. Temp. 14, 618 (1988) [Sov. J. Low Temp. Phys. 14, 340 (1988)].

16. J.E. Lennard-Jones and A.F. Devonshire, Proc. R. Soc. London A163, 53 (1937).

17. Cryocrystals, B.I. Verkin and A.F. Prikhot'ko (eds.), Naukova Dumka, Kiev (1983).

18. V. Kiryukhin, E.P. Bernard, V.V. Khmelenko, R.E. Boltnev, N.V. Krainyukova, and D.M. Lee, Phys. Rev. Lett. 98, 195506 (2007).

19. N.V. Krainyukova, J. Low Temp. Phys. 150, 317 (2008).

20. N. Krainyukova and V. Kraynyukov, J. Phys. CS150, 032047 (2009). 